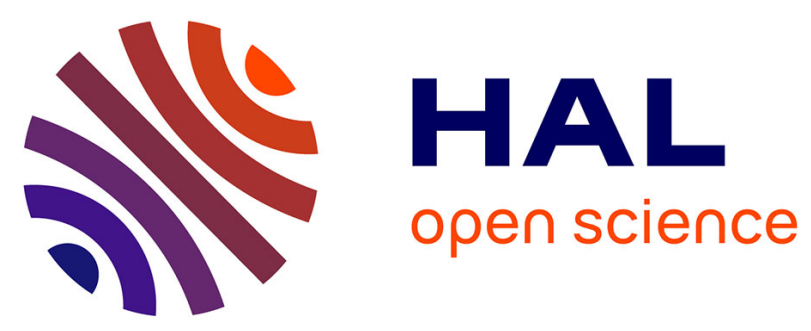

\title{
Spin transfer torque magnetic random-access memory: Towards sub-10 nm devices
}

\author{
N. Perrissin, S. Lequeux, N. Strelkov, L. Vila, L Buda-Prejbeanu, S. Auffret, \\ R. Sousa, I. L Prejbeanu, B. Dieny
}

\section{- To cite this version:}

N. Perrissin, S. Lequeux, N. Strelkov, L. Vila, L Buda-Prejbeanu, et al.. Spin transfer torque magnetic random-access memory: Towards sub-10 nm devices. 2018 International Conference on IC Design \& Technology (ICICDT 2018), Jun 2018, Otrante, Italy. 10.1109/ICICDT.2018.8399772 . hal-01934505

\section{HAL Id: hal-01934505 \\ https://hal.science/hal-01934505}

Submitted on 26 Nov 2018

HAL is a multi-disciplinary open access archive for the deposit and dissemination of scientific research documents, whether they are published or not. The documents may come from teaching and research institutions in France or abroad, or from public or private research centers.
L'archive ouverte pluridisciplinaire HAL, est destinée au dépôt et à la diffusion de documents scientifiques de niveau recherche, publiés ou non, émanant des établissements d'enseignement et de recherche français ou étrangers, des laboratoires publics ou privés. 


\section{Spin transfer torque magnetic random-access memory: Towards sub-10 nm devices}

$\underline{\text { N. Perrissin }}^{1}$, S. Lequeux ${ }^{1}$, N. Strelkov ${ }^{1,2}$, L. Vila ${ }^{1}$, L. Buda-Prejbeanu ${ }^{1}$, S. Auffret ${ }^{1}$, R.C. Sousa $^{1}$, I.L. Prejbeanu ${ }^{1}$, B. Dieny ${ }^{1}$

(1) Univ. Grenoble Alpes, CEA, CNRS, Grenoble INP*, INAC-SPINTEC, 38000 Grenoble, France

(2) Department of Physics, Lomonosov Moscow State University, Moscow 119991, Russia

*Institute of Engineering Univ. Grenoble Alpes

Contact email: bernard.dieny@cea.fr

Magnetic Random-Access Memory (MRAM) is a non-volatile class of solid-state storage device where the information is stored in the magnetic state of a ferromagnetic layer. Microelectronic industry has recently shown a strong interest for MRAM as they are very promising for embedded RAM applications and particularly embedded FLASH replacement ${ }^{1-}$ ${ }^{6}$. The main building bloc of an MRAM is a trilayer $\mathrm{FM}_{1} / \mathrm{I} / \mathrm{FM}_{2}$ (Fig. 1.a) named magnetic tunnel junction $(\mathrm{MTJ})^{7}$, where $\mathrm{FM}_{1(2)}$ refer to ferromagnetic layers and $\mathrm{I}$ to a thin insulating tunnel barrier $(\sim 1.2 \mathrm{~nm})$. Nowadays researches are mainly focused on perpendicularly $(\mathrm{p})$ magnetized tunnel junctions ${ }^{8-11}$ written by spin transfer torque (STT) ${ }^{12,13}$, where the perpendicular axis is defined by the growth direction. $\mathrm{FM}_{1}$ is called the reference layer, its magnetization is pinned in one specific direction (for example, the up direction). $\mathrm{FM}_{2}$ is called the storage layer or free layer, its magnetization is free to be moved between its two stable states (up and down). The resistance of the stack depends on the relative orientation of the two magnetizations. The parallel (P) state (up/up) is a low resistance state while the antiparallel (AP) state (up/down) is a high resistance state, thus respectively coding the " 0 " and " 1 " of the binary logic. The tunneling magnetoresistance (TMR) is defined by the ratio $T M R=\frac{R_{A P}-R_{P}}{R_{P}}$. The $\mathrm{P}$ and AP states are separated by an energy barrier $\mathrm{E}_{\mathrm{b}}$, used to define the thermal stability of the memory $\Delta=\mathrm{E}_{\mathrm{b}} / \mathrm{k}_{\mathrm{B}} \mathrm{T}$ with $\mathrm{k}_{\mathrm{B}} \mathrm{T}$ the thermal energy (Fig. 1.b). For practical applications, one aims for $\Delta$ values from 60 to $100^{14,15}$.

In STT-MRAM, the storage layer has commonly an almost cylindrical shape of diameter $\mathrm{D}$ and thickness L. When the memory cell is sufficiently small to behave in a macrospin manner (typically below 20nm in diameter), its stability is given by Eq. 1, where $\mu_{0}$ is the vacuum permeability, A the storage layer area and $\mathrm{M}_{\mathrm{S}}$ the saturation magnetization. The first term inside the bracket is the shape anisotropy, where $\mathrm{N}_{\mathrm{xx}}$ and $\mathrm{N}_{\mathrm{zz}}$ are the demagnetizing factors. The second and third terms respectively represent the interfacial anisotropy arising at the $\mathrm{FeCoB} / \mathrm{MgO}$ interface and a possible uniaxial bulk anisotropy of magnetocrystalline or magneto-elastic origin. The critical voltage $\mathrm{V}_{\mathrm{c}}$ is defined as the minimum voltage required to write the memory state at $0 \mathrm{~K}$ (Eq. 2), where RA is simply the resistance times area product of the memory point. The write voltage at non-zero temperature can be lower than $\mathrm{V}_{\mathrm{c}}$ and depends on the pulse duration ${ }^{14}$. 


$$
\begin{gathered}
\Delta=\frac{\mathrm{E}_{\mathrm{B}}}{\mathrm{k}_{\mathrm{B}} \mathrm{T}}=\frac{\mathrm{A}}{\mathrm{k}_{\mathrm{B}} \mathrm{T}}\left[\frac{1}{2} \mu_{0} \mathrm{M}_{\mathrm{S}}^{2} \mathrm{~L}\left(\mathrm{~N}_{\mathrm{xx}}-\mathrm{N}_{\mathrm{zz}}\right)+\mathrm{K}_{\mathrm{s}}+\mathrm{K}_{\mathrm{u}} \mathrm{L}\right] \\
\mathrm{V}_{\mathrm{c}}=\frac{4 \mathrm{e}}{\hbar} \frac{\alpha \mathrm{RA}}{\eta} \frac{\mathrm{k}_{\mathrm{B}} \mathrm{T} \Delta}{\mathrm{A}}
\end{gathered}
$$

In conventional p-MTJ, as the thickness of the free layer $(\simeq 1.4 \mathrm{~nm})$ is much lower than its diameter ( $>30 \mathrm{~nm}$ ), so that $N_{x x} \sim 0, N_{z z} \sim 1$. The shape anisotropy (first term in Eq.1) thus favors in-plane anisotropy. The perpendicular magnetization is then obtained thanks to a strong interfacial anisotropy at the FM/I interface which overcomes the in-plane shape anisotropy. The MTJ stack comprises the central block: $\mathrm{FeCoB}(\sim 1.3 \mathrm{~nm}) / \mathrm{MgO}(\sim 1.2 \mathrm{~nm}) / \mathrm{FeCoB}(\sim 1.4 \mathrm{~nm})$ which combines several advantages: After annealing, it provides a good crystallographic structure match between the $\mathrm{FeCo}(001)$ magnetic electrode and the $\mathrm{MgO}(001)$ tunnel barrier ${ }^{16}$, a quite low Gilbert damping constant ${ }^{17,18}$, a strong interfacial anisotropy ${ }^{19,20}$ and a large spin polarization leading to a high TMR ratio ${ }^{11,21}$. However, at sub-20nm dimensions (macrospin behavior), $\Delta$ scales with $\mathrm{D}^{2}$, inevitably leading to excessively low values of $\Delta$ at small diameters meaning too high bit error rate in standby or during read. In conventional STT-MRAM, one can insure $\Delta>60$ down to diameters of around $20 \mathrm{~nm}{ }^{22}$. It can be improved by capping the free layer with another $\mathrm{MgO}$ layer to increase the total interfacial anisotropy. This enables to achieve $\Delta>60$ down to diameters of $\sim 15 \mathrm{~nm}^{23,24}$.

To go to even smaller diameters while maintaining sufficiently high values of $\Delta$, we propose to take advantage of a perpendicular shape anisotropy (PSA) by patterning the storage layer with an aspect ratio L/D of the order or greater than one. By doing this, the shape anisotropy becomes positive and no longer fights against the interfacial anisotropy. To keep good interfacial properties (large TMR and large interfacial anisotropy), one can keep a thin FeCoB layer at the interface with the barrier, then deposit a thick FM layer with low Gilbert damping on top. This approach allows to have both good interfacial properties and to give to the storage layer the desired properties (low damping, weak thermal variation of anisotropy and TMR, etc). Let us for example consider the case of $\mathrm{MgO} / \mathrm{FeCoB}(1.4 \mathrm{~nm}) / \mathrm{Co}(\mathrm{L}-1.4 \mathrm{~nm})$ with a total thickness L (Fig. 2). The smallest thicknesses correspond to the conventional p-STTMRAM regime $(\mathrm{L}<2 \mathrm{~nm}$ ) while the larger ones correspond to the novel PSA-STT-MRAM regime $(\mathrm{t}>3 \mathrm{~nm})$. The diagram clearly illustrates the inability of $\mathrm{p}$-STT-MRAM to maintain $\Delta$ $>60$ at sub-20 nm nodes. On the other hand, once a thick Co layer is added on top of the storage layer, a strong perpendicular anisotropy can be recovered originating from the shape itself. By changing the thickness and/or the diameter of the storage layer, one can tune the stability over a very wide range, even for sub-10 nm nodes. For this example, a stability of $\Delta=60$ can be achieved down to a diameter of $4 \mathrm{~nm}$ with a total storage layer thickness of $32 \mathrm{~nm}$. We superimpose to the stability diagram a boundary evaluated by micromagnetic simulations, indicating the validity range of Eq. 1 . Finally, we also superimpose some iso- $V_{c} / \alpha$ RA lines to emphasize the need of low RA tunnel junctions. Indeed, by considering a write voltage of $V_{c}=$ $0.5 \mathrm{~V}$ and a damping constant $\alpha=0.01$, one needs a RA of $1 \Omega \mu^{2}$ to switch a Co-based PSA-STT-MRAM with $\mathrm{D}=7 \mathrm{~nm}, \mathrm{~L}=16 \mathrm{~nm}(\Rightarrow \Delta=60)$. The smaller the diameter, the smaller $\alpha$ and RA have to be.

Co-based and FeNi-based PSA-STT-MRAM with thickness of $60 \mathrm{~nm}$ have been patterned by IBE in a two-step process. The storage layer was first etched vertically thanks to a hard mask of Ta with $\mathrm{D}=30 \mathrm{~nm}$. The pillar was then trimmed to sub-20 nm diameters by etching at a 
grazing angle. The lower diameter observed after trimming is $8.1 \mathrm{~nm}$. Hysteresis resistance versus magnetic field loops (Fig. 3) show two well defined resistance states with TMR values around $50 \%$ and $\Delta$ values coherent with micromagnetic simulations.

(a)

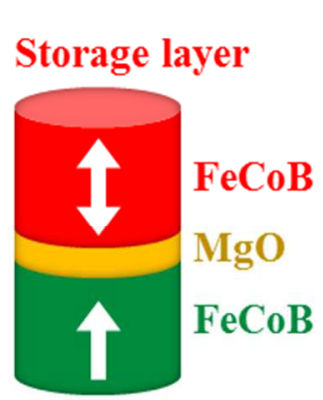

Reference layer (b)

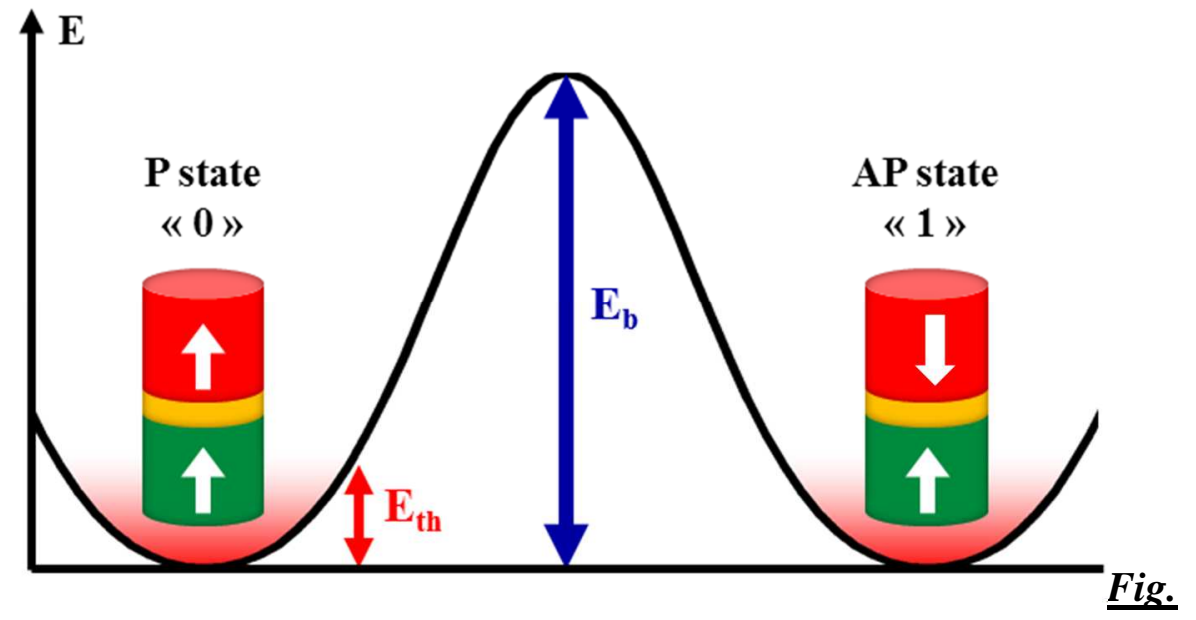

1. (a) Sketch of a conventional p-MTJ. In red the storage layer FeCoB(1.4 nm) with two stable states highlighted by the white double-arrow, in yellow the $\mathrm{MgO}(1.2 \mathrm{~nm})$ tunnel barrier and in green the reference layer $\mathrm{FeCoB}(1.3 \mathrm{~nm})$ with the magnetization pinned in one direction highlighted by the white single-arrow. (b) Energy profile of the p-MTJ versus the orientation of the magnetization of the storage layer. The stability factor is defined by the ratio $\Delta=\frac{E_{b}}{k_{B} T}$.

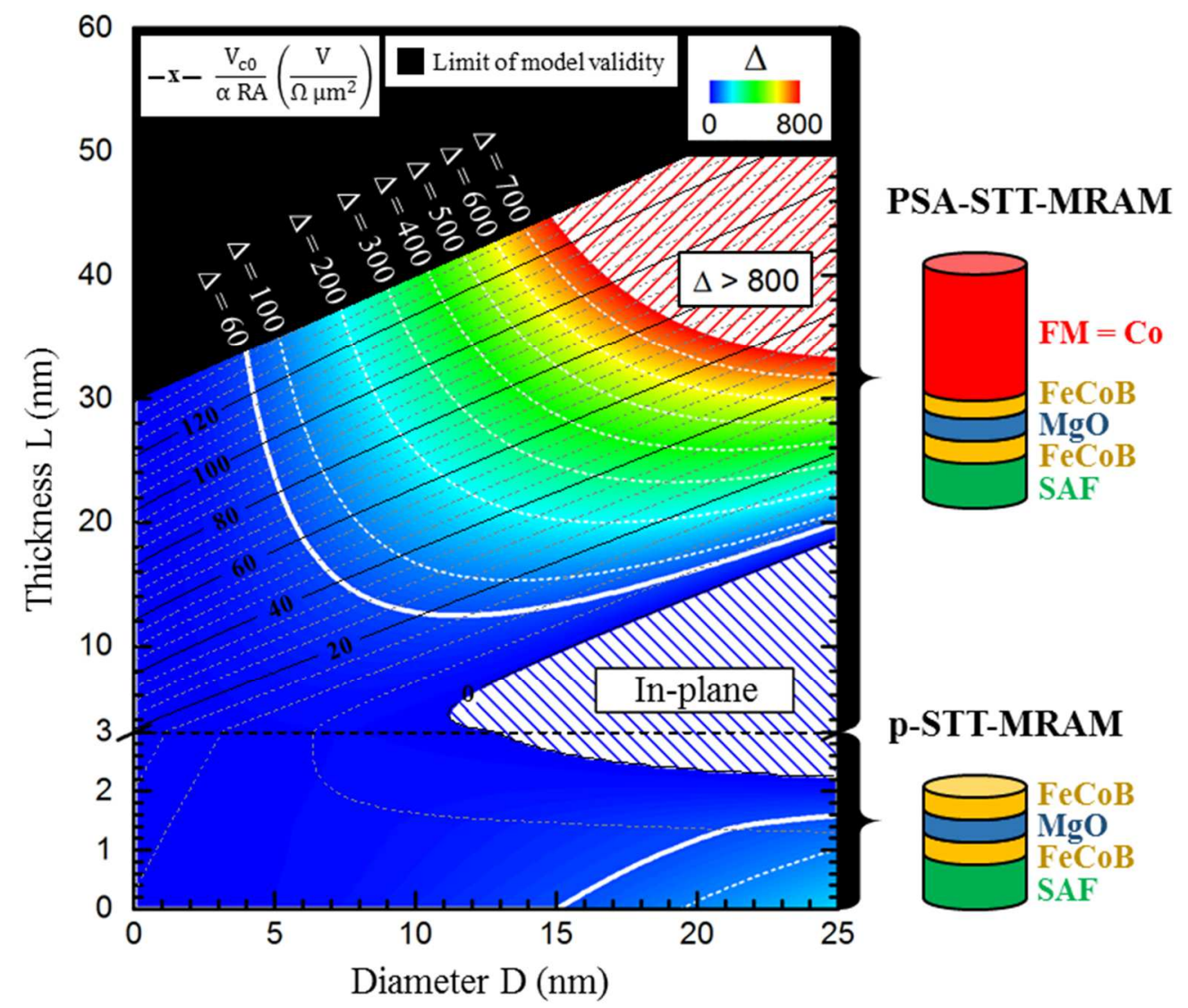

Fig 2. Stability diagram of a cylindrical storage layer made of $\mathrm{FeCoB}(1.4 \mathrm{~nm}) / \mathrm{Co}(\mathrm{L}-1.4 \mathrm{~nm})$ versus its total thickness $(L)$ and diameter $(D)$, at room temperature $(300 \mathrm{~K})$. Below a total 
thickness of $1.4 \mathrm{~nm}$, the storage layer is supposed to only consist of $\mathrm{FeCoB}$ with $\mathrm{M}_{S}^{\mathrm{FeCoB}}=1.0$ $10^{6} \mathrm{~A} / \mathrm{m}$ and $K_{S}^{\mathrm{FeCoB}}=1.4 \mathrm{~mJ} / \mathrm{m}^{2}$. Above $1.4 \mathrm{~nm}$, the storage layer consists of $\mathrm{FeCoB}(1.4 \mathrm{~nm})$ $/ C o(L-1.4 \mathrm{~nm})$, with $M_{S}^{C o}=1.44610^{6} \mathrm{~A} / \mathrm{m}$ and $K_{u}^{C o}=0 \mathrm{~J} / \mathrm{m}^{3}$. The vertical axis is cut in two parts to better show the first $3 \mathrm{~nm}$, corresponding to the standard p-STT-MRAM regime. The blue dashed area represents the in-plane regime. The red dashed area represents the region where $\Delta$ is greater than 800. The smaller thicknesses correspond to the standard p-STT-MRAM regime while the larger ones correspond to the PSA-STT-MRAM regime, as described by the sketches on the right of the diagram. The black area represents the region where Eq. 1 and 2 are no longer verified. Some iso- $\Delta$ lines are highlighted in white and in particular $\Delta=60$ in bold white. Some iso- $V_{c} / \alpha R A$ lines are highlighted in black and grey with $\eta=0.88$. For example $V_{c}=1$ $V, \alpha=0.01$ and $R A=5 \Omega \cdot \mu m^{2}$ is represented by the iso- $V_{c} / \alpha R A=20 \mathrm{~V} \cdot\left(\Omega \cdot \mu \mathrm{m}^{2}\right)^{-1}$.

(a)

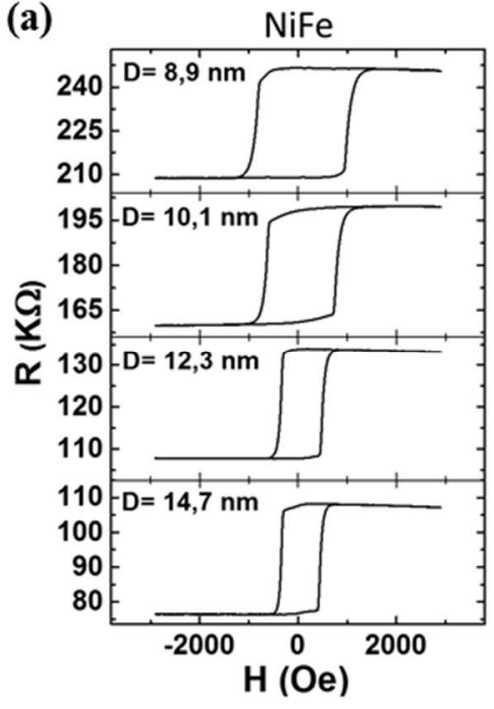

(b)

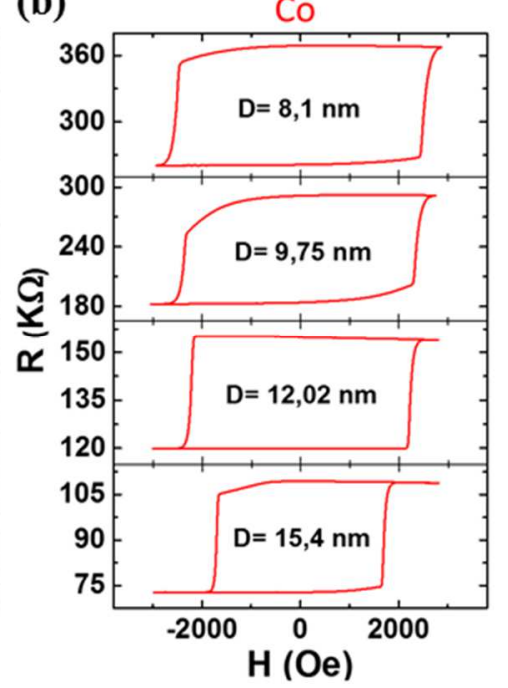

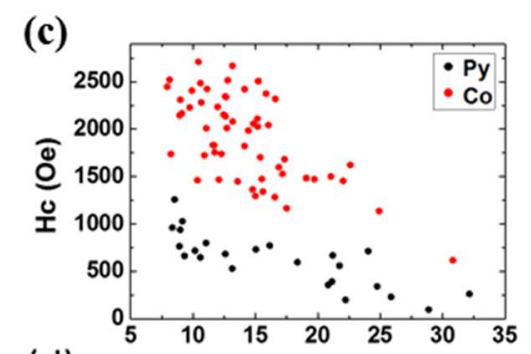

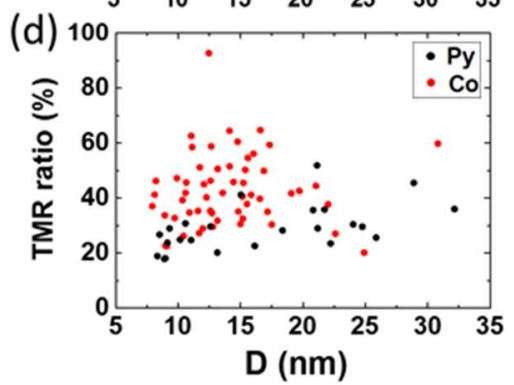

Fig. 3. (a), (b)Resistance $R$ versus perpendicular field for MTJ of various diameters $D$ in the cases of a $60 \mathrm{~nm}$ thick storage layer of NiFe and Co respectively. (c) Distribution of coercive fields $H_{c}$ as a function of $D$ for NiFe (black circles) and Co (red circles) storage layer. (d) Distribution of the TMR ratio as a function of D for NiFe (black circles) and Co (red circles) storage layer.

This work was funded by ERC Adv grant MAGICAL 669204.

The authors would like to thank Jannier Roiz for fruitful discussions and valuable suggestions.

1. Kent, A. D. Nat. Mater. 9, 699 (2010)

2. Rizzo, N. D. et al. IEEE Trans. Magn. 49, 44414446 (2013)

3. Ikeda, S. et al. Nat. Mater. 9, 721-724 (2010)

4. Rodmacq, B.et al. J. Appl. Phys. 93, 7513-7515 (2003)

5. Khvalkovskiy, A. V. et al. 46, 139601 (2013)

6. Nishimura, N. et al. J. Appl. Phys. 91, 52465249 (2002)

7. Julliere, M. Phys. Lett. A 54, 225-226 (1975)

8. Monso, S. et al. Appl. Phys. Lett. 80, 4157-4159 (2002)

9. Yuasa, S. et al. Nat. Mater. 3, 868-871 (2004)

10. Parkin, S. S. P. et al. Nat. Mater. 3, 862-867 (2004)
11. Ikeda, S. et al. Appl. Phys. Lett. 93, 082508 (2008)

12. Berger, L. Phys. Rev. B 54, 9353 (1996)

13. Slonczewski, J. C. J. Magn. Magn. Mater. 159, L1-L7 (1996)

14. Dieny, B. et al. Introduction to magnetic random-access memory. (wiley, 2016)

15. Apalkov, D. et al. Proc. IEEE 104, 1796-1830 (2016)

16. Yakushiji, K. et al. Appl. Phys. Express 3, 053003 (2010)

17. Schoen, M. A. W. et al. Nat. Phys. 12, 839-842 (2016)

18. Mankovsky, S. et al. Phys. Rev. B 87, (2013)

19. Krounbi, M. et al. ECS Trans. 69, 119-126 (2015) 
20. Manchon, A. et al. J. Appl. Phys. 104, 043914 (2008)

21. Lee, Y. M. et al. Appl. Phys. Lett. 90, 212507 (2007)
22. Sato, H. et al. Appl. Phys. Lett. 105, 062403 (2014)

23. $\mathrm{Hu}, \mathrm{G}$. et al. IEEE International Electron Devices Meeting (IEDM) 26.3.1-26.3.4 (2015)

24. Cuchet, L. et al. Sci. Rep. 6, srep21246 (2016) 